\title{
The Anesthetic Effect of Dexmedetomidine on ERCP of Senile Patients
}

\author{
Feng Liang, Fang-lei Han and Shuang Qi ${ }^{a}$ \\ China - Japan Union Hospital of Jilin University
}

\begin{abstract}
Objective: to discuss the effectiveness and security of Dexmedetomidine's conscious-sedation application in ERCP of senile patients. Method: selecting 80 senile patients performed with ERCP and randomly dividing them into Dexmedetomidine group (group D) and normal saline group (group C) with 40 cases in each group. Pumping $0.5 \sim 0.8 \mathrm{ug} / \mathrm{kg}$ Dexmedetomidine through the vein 15 min after surgery while pumping isodose NS to group C at the same time. Results: both the two groups completed the ERCP treatment. The tolerance of group $\mathrm{C}$ is poorer with more adverse reactions; the operating time of group $\mathrm{D}$ is shorter, but the difference has no statistical significance. During the operation, compared with group $\mathrm{D}$, the MBP and HR value of group $C$ rose obviously with significant difference $(\mathrm{P}<0.05)$ while the MBP has no obvious change within the group; HR declined sharply after pump injection $(\mathrm{P}<$ 0.05 ), but the value is within the normal range. The contrast of SPO2 and RR within and between the two groups have no significant difference. Conclusion: applying Dexmedetomidine for conscioussedation in senile patients' ERCP leads to hemodynamic stability and no respiratory depression with less adverse reactions. The application during the perioperative period is effective, safe and comfortable, worthy of popularization and application in clinic.
\end{abstract}

\section{Instruction}

With the unceasing development of ERCP technology, ERCP has become an important diagnosis and treatment of pancreatic disease, but during the treatment, patients will suffer abdominal pain, nausea and vomiting, so the intense discomfort will make patients unable to actively cooperate with the operation. Besides, the majority of ERCP group are senile patients. The stress of operation can cause threat to the life of senile patients. Therefore, it is necessary to adopt painless technique and strengthen monitoring. But ERCP patients are in prone position with difficult management breathing. The most of the initial pain-free technology adopts trachea cannula with general anesthesia. The anesthesia is costly with complicated operation and slow postoperative recovery. In order to improve the treatment quality and guarantee the security of senile patients during the perioperative period, our hospital adopts Dexmedetomidine for conscious sedation and observes the clinical effect so as to evaluate the effectiveness and security of its application in ERCP of senile patients.

\section{Materials and Methods}

${ }^{a}$ Corresponding author: qishuang@jlu.edu.cn 


\subsection{General data}

Selecting 80 senile patients performed with ERCP at the age from 55-75 years old and weighing 50-75 $\mathrm{kg}$, including 42 males and 38 females; ASA is in II-III level. They had no heart and respiratory system disease, incomplete liver and kidney function or drug allergy history. Randomly dividing the patients into Dexmedetomidine group (group D) and NS group (group C), namely 40 cases in each group.

\subsection{Methods}

Preoperative patients took $10 \mathrm{ml} 2 \%$ lidocaine mucilage orally. After the pharyngeal numbness, their ECG, NBP, SPO2 and RR would get routinely monitored. Patients added oxygen reservation during surgery by 3 -min oxygen uptake through a nasal mask. Pumping 0.5 0.8ug $/ \mathrm{kg}$ Dexmedetomidine through the vein to group D in 15 minutes and observing the patient's condition. When patients reacted slowly and went into the state of sleepiness but could still be awakened, then it is the timing to start operation. The patients were sent to the viewing room after operation. They wouldn't be sent back to the ward until complete revival; group $\mathrm{C}$ would be operated after pumping isodose NS at the same time.

\subsection{Observing Indexes}

Recording the $\mathrm{HR}, \mathrm{MBP}, \mathrm{SPO}_{2}$ and $\mathrm{RR}$ of the two groups respectively when entering the operating room ( $\mathrm{t} 0)$, the pumping moment ( $\mathrm{t} 1)$, the end of pumping ( $\mathrm{t} 2)$, the endoscopic insertion moment ( $\mathrm{t} 3$ ), the beginning of angiography ( $\mathrm{t} 4)$ and the withdrawal moment of endoscope; recording adverse reactions such as bucking, nausea, vomiting, restlessness, etc; recording the temporal summation (operating time) from endoscopic insertion to withdrawal.

\subsection{Statistics processing}

Using $\mathrm{t}$ to test measurement data and adopting $\chi^{2}$ to verify enumeration data. $\mathrm{P}<0.05$ means the difference has statistical significance.

\section{Results}

The differences of general data between groups had no statistical significance. They all completed EVL treatment. The tolerance of group $\mathrm{C}$ resistance was poorer with more adverse reactions; the operating time of group $\mathrm{D}$ was shorter than group $\mathrm{C}$, but there was no statistically significant difference between the two groups (table 1). Comparing with the time point of $t_{0}$, the MBP and HR within group $C$ and group D was significantly increased in the process of operation $\left(t_{3} \sim t_{5}\right)$, so the difference was significant $(\mathrm{P}<0.05)$; the MBP of group $\mathrm{D}$ had no obvious change comparing with the values of other 5 time points; HR declined significantly after pumping ( $\mathrm{t} 2 \sim \mathrm{t5})(\mathrm{P}<0.05)$, but the values were all within the normal range. There was no significant difference of $\mathrm{SPO}_{2}$ and $\mathrm{RR}$ value between groups and within groups (table 2).

Table 1. the comparison of adverse reactions and operation time of the two groups of patients $(n=40)$

\begin{tabular}{cccccc}
\hline Groups & Nausea & Emesis & Bucking & Dysphoria & Operation time \\
\hline Group D & 5 & 0 & 0 & 0 & $9.2 \pm 2.4$ \\
Group C & $30(75 \%)$ & 4 & $8(20 \%)$ & 3 & $11.2 \pm 1.5$ \\
\hline
\end{tabular}


Table 2. the comparison of the changes of MBP, HR, $\mathrm{SPO}_{2}$ and $\mathrm{RR}$ of the two groups of patients

\begin{tabular}{|c|c|c|c|c|c|c|c|}
\hline $\begin{array}{c}\text { Index } \\
\text { es }\end{array}$ & Groups & to & $\mathrm{t} 1$ & $\mathrm{t} 2$ & t3 & $\mathrm{t} 4$ & t5 \\
\hline \multirow[b]{2}{*}{ MAP } & Group D & $93.2 \pm 2.4$ & $96.7 \pm 2.1$ & $95.2 \pm 2.1$ & $97.2 \pm 1.9$ & $98.2 \pm 2.4$ & $97.2 \pm 2.9$ \\
\hline & Group C & $96.3 \pm 2.5$ & $97.1 \pm 2.6$ & $96.8 \pm 2.0$ & $106.6 \pm 3.7^{*}$ & $103.5 \pm 3.6^{*}$ & $104.2 \pm 4.2^{*}$ \\
\hline \multirow{2}{*}{ HR } & Group D & $83.5 \pm 11.7$ & $83.7 \pm 12.5$ & $72.5 \pm 9.7^{*}$ & $72.4 \pm 9.4^{*}$ & $73.5 \pm 8.7^{*}$ & $74.5 \pm 8.8^{*}$ \\
\hline & Group C & $84.5 \pm 11.9$ & $84.3 \pm 11.9$ & $83.5 \pm 11.9$ & $95.6 \pm 8.2^{\#}$ & $96.7 \pm 8.5^{\#}$ & $97.5 \pm 8.7^{\#}$ \\
\hline \multirow{2}{*}{$\mathrm{SPO} 2$} & Group D & $96.5 \pm 2.5$ & $96.5 \pm 2.5$ & $96.4 \pm 2.6$ & $96.5 \pm 2.5$ & $95.6 \pm 2.4$ & $96.7 \pm 2.1$ \\
\hline & Group C & $96.7 \pm 2.3$ & $97.2 \pm 2.8$ & $99.8 \pm 2.2$ & $95.9 \pm 2.1$ & $96.5 \pm 2.4$ & $96.5 \pm 2.4$ \\
\hline \multirow{2}{*}{$\mathrm{RR}$} & Group D & $13.7 \pm 6.2$ & $13.2 \pm 6.2$ & $11.4 \pm 7.2$ & $12.5 \pm 6.7$ & $12.5 \pm 6.3$ & $12.9 \pm 6.1$ \\
\hline & Group C & $13.1 \pm 6.3$ & $13.1 \pm 6.3$ & $13.9 \pm 7.8$ & $17.2 \pm 6.2^{\triangle}$ & $16.8 \pm 6.6^{\triangle}$ & $13.2 \pm 6.2$ \\
\hline
\end{tabular}

Comparing the data of $\mathrm{t}_{3}-\mathrm{t}_{5}$ of group $\mathrm{C}$ with group $\mathrm{D}: \mathrm{P}^{*}<0.05 ; \mathrm{P}^{\#}<0.05 ; \mathrm{P}^{\triangle}<0.05$;

Comparing he data of $\mathrm{t}_{2}-\mathrm{t}_{5}$ of group $\mathrm{D}$ with $\mathrm{t} 0$ : $\mathrm{P}^{*}<0.05$.

\section{Discussions}

Comfortable and secure medical service is the development trend of clinical medicine in recent years. Though ERCP can be performed under local anesthesia, but senile patients' tolerance of strong stress is poor, so cardiocerebral events are likely to be induced; adverse reactions such as bucking, nausea and vomiting, dysphoria and so on often interfere with the operation and increase the risk of bleeding ${ }^{[3]}$. This study found that $75 \%$ of senile patients of group $\mathrm{C}$ suffered different degrees of nausea and vomiting while $20 \%$ of them appeared bucking. The RR, HR and blood pressure of each time point during the operation rose obviously comparing with the moment when they entered the operating room, which suggested that performing ERCP on senile patients on the condition of topical anesthesia had certain risks.

Painless microscopy is usually completed under intravenous anesthesia. Using intravenous anesthetics or analgesia medicine often causes swallowing, bucking and other protective areflexia, so aspiration is easy to happen and difficult to deal with. In addition, the commonly used intravenous anesthetics, such as propofol and fentany, have some inhibiting effect on angiocarpy and respiratory system. The haemodynamics of most elderly people is not stable. A small amount of sedative and analgesic drugs may cause obvious inhibition of angiocarpy and respiratory functions, so such operations adopting intravenous anesthetics on senile patients should be performed particularly cautious.

Conscious sedation is a moderate level of sedation, whose level is lower than intravenous anesthesia. The patients are in the state of sleeping and they can respond to language and tactile stimulus with deglutition reflex. Their respiratory and circulatory functions are stable ${ }^{[4]}$. Dexmedetomidine is a kind of high-selectivity $a^{2}$ adrenergic agonist, which can obviously reduce the sympathetic reactions to stress. This kind of sedation is similar to natural sleep. Unlike other sedatives, it acts on the brainstem nucleus ceruleus, so no respiratory inhibition will appear and certain swallowing function can be maintained. This study found that the patients of group D suffered little change of haemodynamics at each time point with no respiratory depression or less adverse reactions, so the sedation level reaches the level of the security, comfort and ease of operation.

Above all, applying Dexmedetomidine in ERCP for senile patients for conscious sedation is effective, secure and comfortable during the perioperative period, so it deserves popularization and application in clinic.

\section{References}

1. Schepis F,Camma C,Niceforo D, et al. Which patients with cirrhosis should undergo endoscpic screening for esophageal varices detection[J].Hepatology,2001,33:333 338.

2. Williams S GJ, Westahy D.Recent advances in the endoscopic management of variceal bleeding[J].Cut,1996,36(6):647. 
3. Zigang $\mathrm{Wu}$, Qiusheng Zhang, Huabin Quan. Comparison of four therapeutic methods in the treatment of esophageal varices bleeding [J]. Chinese Journal of Digestive Endoscopy, 2000, 339-341. Chinese.

4. Faigel DO,Baron TH,Goldstein JL,et a1.Guidelines for the use of deep sedation and anesthesia for GI endoscopy[J]. Gastmintest Endosc,2002,56: 613 617.

5. Hao Pei. Pharmacological effects of dexmedetomidine hydrochloride and its clinical application [J]. Herald of Medicine, 2012, 12:33 34. Chinese. 